\title{
Recent Advances in Microwave-based Dielectric Spectroscopy at the Cellular Level for Cancer Investigations
}

\author{
K. Grenier, Member, IEEE, D. Dubuc, Member, IEEE, T. Chen, F. Artis, T. Chretiennot, \\ M. Poupot, and J-J. Fournié
}

\begin{abstract}
Cancer remains a leading cause of death in the world. To overcome this problem, it is necessary to develop new analysis tools, in complementarity to existing ones, to enable the early diagnostic of the diseases, personalized treatment, and further fundamental cancer mechanisms understanding. In this context, micro and millimeterwave-based dielectric spectroscopy performed at the cellular and molecular levels is progressively emerging, as it permits the non-invasive and real time probing of cells in their culture biological medium. The recent advances of this topic are given in this paper with a specific highlight of its various assets.
\end{abstract}

Index Terms-Dielectric spectroscopy, microwave, biology, cancer, cells

\section{INTRODUCTION}

$\mathrm{T}$ HE biological and medical domains have potential for massive modifications due to the strong development of microfluidics, as it corresponds to the appropriate size to reach biomolecules and cells properties. Micro and nanotechnologies offer well-controlled dimensions and fluidic volumes (as low as microliters and even smaller!) at a quite low cost and high fabrication volumes without the manualhandling drawbacks [1]. It is now possible to reproducibly engineer cell microenvironment at the cellular resolution and to mimic complex biochemistries (cells interaction, gradients, cells contacts...). Cells culture, sorting, handling and transportation [2], as well as addition of chemicals, combined with single to arrays arrangements for high throughput analysis [3]-[4] have been demonstrated and present great interests for future cancer investigations [5]. Early cancer diagnostic, personalized treatments with the possible preevaluation of chemotherapies and fundamental researches toward a better understanding of cancer mechanisms constitute strong challenges to the biologists and physicians communities.

The French RITC Foundation Grant supported a part of the included work.

K. Genier, D. Dubuc T. Chen, F. Artis and T. Chretiennot are with the National Scientific Research Center, Laboratory of Analysis and Architecture of Systems, 31400 Toulouse, France (phone: +33 56133 6291; fax: +33 56133 6969; e-mail: grenier@ laas.fr).

M. Poupot and J-J. Fournié are with the Cancer Research Center of Toulouse, Toulouse, 31000 France. (e-mail: poupot@inserm.fr)
One important bottleneck of complete lab-on-a-chips development is the miniaturization of appropriate analyzing systems. The actual and major cellular analysis tools employed by biologists are based on optical detection of fluorescent tags or cell staining, which require cumbersome equipment as well as time-consuming preparation phase. The major problem consists consequently in proposing integrated characterization techniques, which are sensitive and able to operate non-invasively in liquid medium. Intensive researches are consequently on going in order to answer these leitmotivs with optical, electrical - low frequency $(\leq \mathrm{MHz})[6]$ or very high frequency (THz) [7]-[8], and mechanical [9]-[11] solutions in fluidic environment [12].

Among all these sensing methods, the microwave dielectric spectroscopy represents a very promising solution. It benefits from the extensive researches that have been previously realized at tissues and cells suspensions levels for several decades [13]-[15]. The discrimination of tumorous and nontumorous tissues has led to the wide development of breast and liver cancers imaging notably [16]-[20] and lymphatic disease detection [21].

Based on these remarkable demonstrations, the micro and millimeterwave dielectric spectroscopy at the cellular level and for cancer investigations notably is rapidly emerging. This paper consequently focuses on this topic to allow the noninvasive observation of living and, especially of cells and molecules, in liquid at the micrometric scale. After a first section, which reminds some fundamental interaction mechanisms of microwaves with the biological matter, the different assets of the micro and millimeter-wave-based sensing at the molecular and cellular levels are presented.

\section{FUNDAMENTAL INTERACTION MECHANISMS OF MICROWAVES WITH BIOLOGICAL MATTER}

The interaction of electromagnetic waves with biological matter has been well established during the past century with many contributions and notably the ones from Fricke [22], Cole [23], and Schwan [13] up to few GHz. The frequency dependence of the dielectric properties of the biological matter to the applied electric field has been revealed and attributed to 
several polarization phenomena. The broadband investigations of Schwan [13] has led to a clear view of the different dispersions of the relative dielectric constant $\varepsilon$, which occur at specific frequency ranges and are attributed to particular physical phenomena, as indicated in Fig. 1.

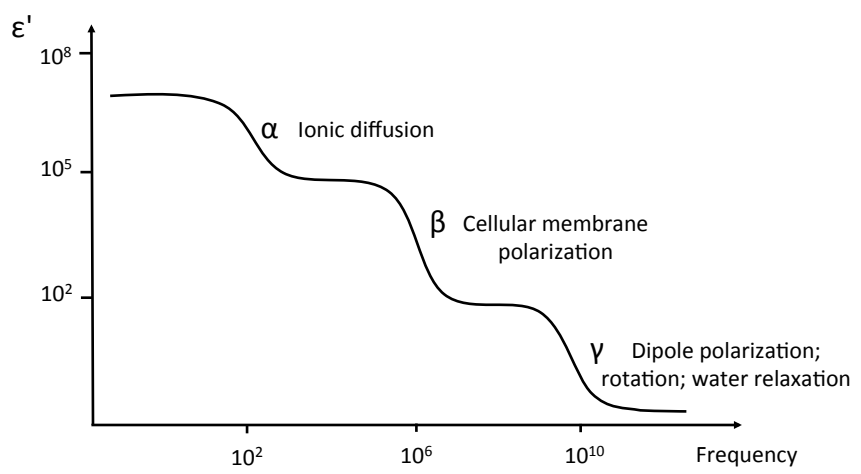

Fig. 1. Relative dielectric constant $\varepsilon$ versus frequency and the three major dispersions.

The first dispersion, named $\alpha$, is attributed to the ionic diffusions, whereas the $\beta$ one located in the $\mathrm{MHz}$ range is related to the polarization of the cellular membrane. By increasing the frequency, the capacitive barrier of the cells membrane may be crossed and waves may penetrate in the cells. The next major relaxation called $\gamma$ dispersion is then situated at microwaves and corresponds mainly to the polarization of the electrical dipole moment of molecules. It includes the relaxation of water molecules, with a corresponding frequency located around $20 \mathrm{GHz}$. A fourth additional and weak dispersion named $\delta$ may also be distinguished between the $\beta$ and $\gamma$ ones in some cases. Further in frequency, in the $\mathrm{THz}$ regime, others mechanisms are also occurring based on vibrational modes and atomic variations [24].

Consequently, the permittivity may often be described by a succession of relaxations, which can be described by

$$
\varepsilon^{*}=\varepsilon_{\infty}+\sum_{i} \frac{\Delta E_{i}}{1+\left(j \omega \frac{1}{f_{i}}\right)^{1-\alpha}}-\frac{j \sigma}{\omega \cdot \varepsilon_{0}},
$$

where $\varepsilon_{\infty}$ refers to the high frequency permittivity, $\omega$ the angular frequency, $\Delta \mathrm{E}_{\mathrm{i}}$ the change in permittivity for each dispersion identified by the subscript $i, f_{i}$ the relaxation frequency corresponding to the maximal value of the imaginary part of permittivity and $\sigma$ the conductivity. The parameter $\alpha$ of (1) is a number between 0 and 1 [13], [23].

The decreases of the relative permittivity are simultaneously accompanied by a peak of the imaginary part of the permittivity at the relaxation frequency.

Even if the polarization phenomena have been well defined on a large frequency range, the exact interaction mechanisms in the micro and millimeterwave frequency ranges $(\gamma$ dispersion) at the cellular and molecular levels are not entirely understood. Some assumptions of mechanisms may nevertheless be made. First should be reminded the large constitution of the living matter with water, and the water relaxation due to its molecular reorientation dynamic is happening in the microwave range, as previously mentioned. Added to the hydrogen bounding ability of water to cells constituents, water surrounded molecules and cells in aqueous based solutions may lead to particular and distinguishable microwave signatures. Other effects may also be due to ions migrations within the cytoplasm.

Besides these mechanisms of interaction, biosensing at micro and millimeterwave for cells investigations presents multiple interests. The $\gamma$ dispersion imposes large detectable variation of permittivity. The associated wave attenuation due to water is manageable. Furthermore, micro and millimeterwaves are classified as non-ionizing radiations, which also constitutes an important feature toward the development of non-invasive investigations of the living.

The use of microtechnologies for both microwave sensing and fluidic handling and preparation of biological samples may now offer further understanding of the electromagnetic interaction with the living at the cellular and molecular levels and contribute to the development of new analyzing tools to biologists.

\section{MICRO AND MILLIMETERWAVE SENSING OF MOLECULES AND CELLS IN LIQUID AT THE MICROSCALE}

The development of microwave-based micrometric biosensors, adapted to the sensing of molecules and cells in liquid, is progressively emerging. Stuchly et al. firstly published it in 1998 [25] with solvents or de-ionized water placed into a reservoir on top of a planar waveguide.

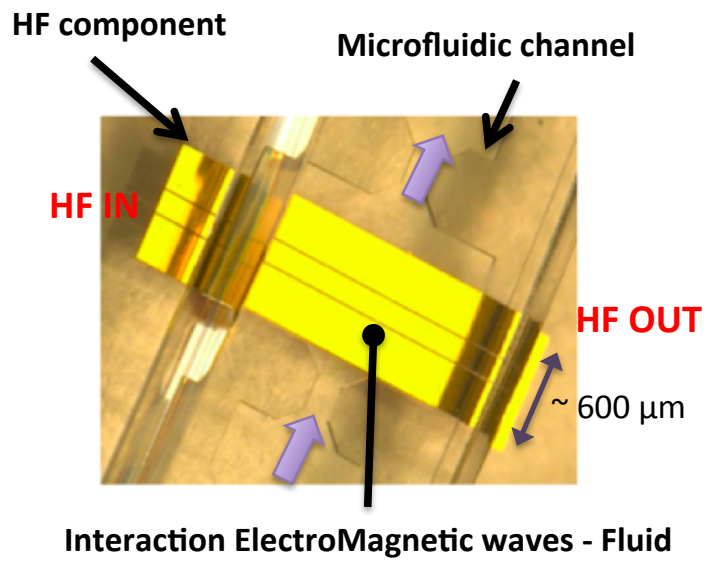

Fig. 2. Miniature fluidic and microwave based biosensor.

Facer et al. in 2001 [26] then demonstrated the broadband detection of hemoglobin and $\lambda$-DNA in liquid, whereas Shaforost et al. in 2009 [27] investigated a resonator configuration for liquids evaluation in the nanoliter range. To circumvent any contamination and evaporation with open 
reservoirs and increase the accuracy, capillaries or microfluidic channels have been applied in whispering-gallery mode resonator [28], coaxial resonator [29], or on top of coplanar lines [30]-[31]. An example is given in Fig. 2 with a miniature fluidic and microwave CPW-based biosensor, which requires microliter volumes of liquid. Such a structure permits the dielectric spectroscopy of various liquids in the micro and millimeterwave ranges. Both real and imaginary parts of the complex relative permittivity may be extracted accurately [31]-[32], as illustrated in Fig. 3 with the microwave signatures of two proteins in aqueous solution, Bovine Albumin Serum (BSA) and saccharose respectively, with a concentration of $100 \mathrm{mg} / \mathrm{ml}$.

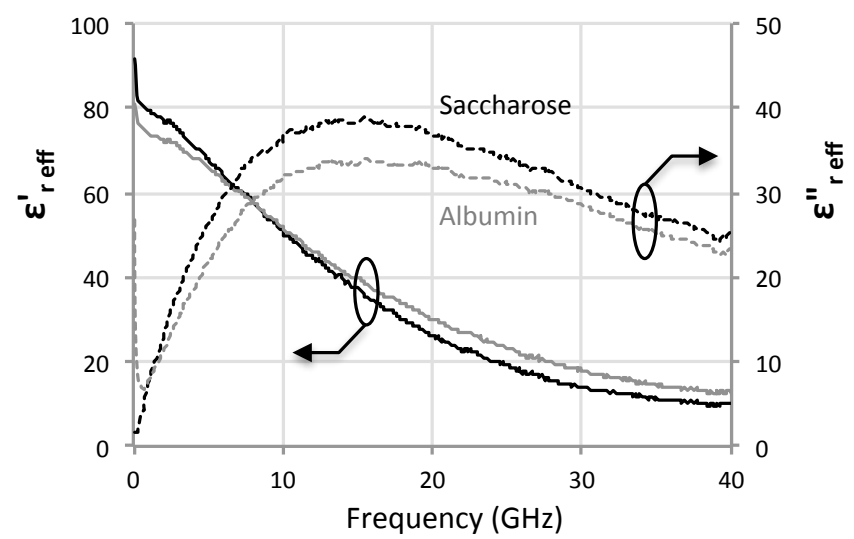

Fig. 3. Real and imaginary parts of the complex permittivity of the $100 \mathrm{mg} / \mathrm{ml}$ Bovine Albumin Serum (in grey) and Saccharose (in black) proteins up to 40 $\mathrm{GHz}$.

The investigation of micro and millimeterwave sensing at the cellular and molecular levels presents several interests, which are illustrated below.

\section{A. Non-invasivity, contact less and label free}

Intrinsically, one strong asset of the microwave detection consists in not requiring direct contact with the sample to analyze, contrary to DC-electrical or mechanical techniques [9]-[10]. This ability minimizes the possible contamination or perturbation of the biological material. The technique may also be marker-less. The addition of chemical substances or particles [33], which may modify the media under study and is time-consuming, is not mandatory.

However, one has to consider other potential side effects on measurement accuracy and biomaterials. At first, microwave energy is absorbed by the matter and converted into heat, due to the molecular movements. Thermal effects may consequently happen and have therefore been appropriately investigated [34] and exploited for hyperthermia therapies notably [14]. However, as the dielectric response of bioliquids is temperature dependent [35]-[38], microwave sensing may be altered. To perform an accurate dielectric spectroscopy on biological cells, the temperature should therefore be carefully controlled.

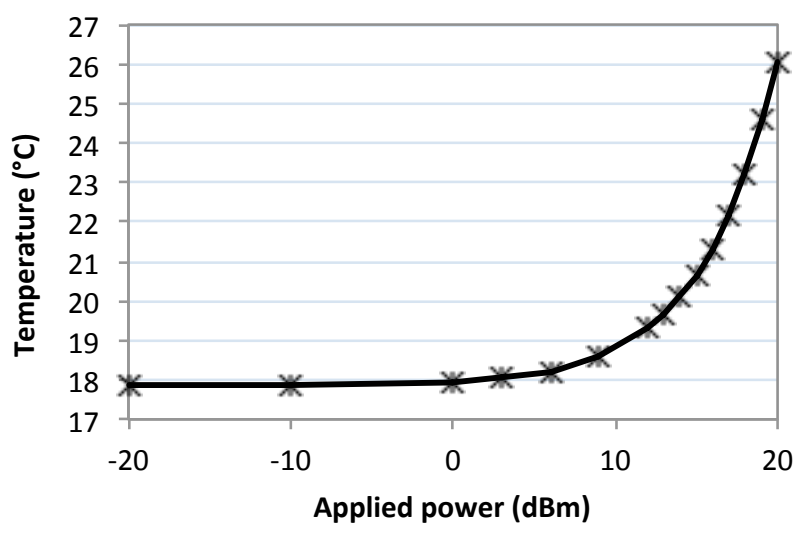

Fig. 4. Thermal impact of power applied at $20 \mathrm{GHz}$ into a 2 microliters reservoir filled with de-ionized water.

Investigation of microwave heating of such microscale microfluidic biosensors (cf. Fig. 2) has to be specifically performed. Even if radio and microwaves are non-invasively used through organs and bodies for implantable wireless medical systems [39], the situation at the microscale may be different, as the bio-objects feature volume to surface ratio few orders of magnitude lower than tissues, organs and bodies. Toward this objective, a 2 microliters fluidic reservoir filled with de-ionized water has been realized on a coplanar line. The temperature has been measured for increasing input powers at $20 \mathrm{GHz}$ with a miniature thermocouple, which was previously calibrated and then immersed in the reservoir. The corresponding results are presented in Fig. 4 and demonstrate no significant temperature increase below a $0 \mathrm{dBm}$ applied power. The choice of very low RF signal powers, $-10 \mathrm{dBm}$ for instance in that case, associated to a thermal control during microwave measurements should consequently be preferred to avoid any thermal interference during dielectric spectroscopy.

Other non-thermal effects on the biological material may also appear and affect different biological aspects, such as DNA [40], enzyme activity [41], and cellular proliferation [42]. Actually, most of these investigations have been realized on large amounts of cells. The development of miniature analyzing tools for biomedical purposes requires now extending these studies to small cells numbers and even down to the single cell level [43]. The proliferation of cells after RF exposure has therefore been evaluated with the reservoir configuration of the component presented in Fig. 2 [44]. Adherent cells of Normal Rat Kidney (NRK) were used and submitted to power of $-10 \mathrm{dBm}$, and $+5 \mathrm{dBm}$. The results are presented in Fig. 5 and demonstrate that there is no significant impact of such power levels on the cells proliferation.

Further investigations with higher power exposure have shown that the cells proliferation is stopped for a power level higher than $+8.6 \mathrm{dBm}$. 


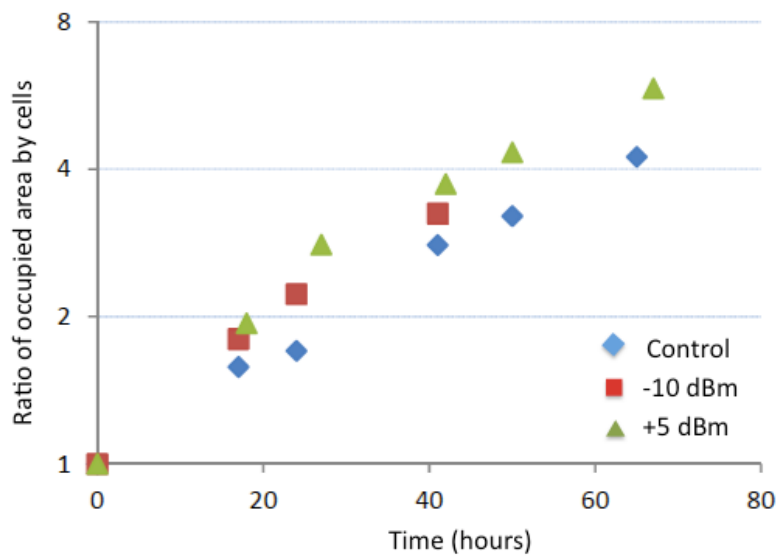

Fig. 5. Impact of applied power at $20 \mathrm{GHz}$ on the cell proliferation rate for $10 \mathrm{dBm}$ and $+5 \mathrm{dBm}$.

The non-invasivity of the electromagnetic waves on cells at the micrometric scale for biomedical investigations has consequently to be carefully checked. Further investigations should be foreseen with other precise and complementary indicators of cells viability.

\section{B. Impact of ionic content}

Besides the non destructive and non-invasivity of micro and millimeterwaves, another advantage should be highlighted. As presented in Fig. 6, the ionic conduction of traditional biological solutions for cells culture does not present any trouble in the microwave range. The conventional salt concentration of $9 \mathrm{~g} / \mathrm{l}$ of typical biological media is not screening the signals for these frequencies. Fig. 6 presents the imaginary part of the relative permittivity of de-ionized water and Roswell Park Memorial Institute (RPMI) biological medium. Losses induced by the salt content of RMPI are drastically increasing at low frequency. On the contrary, salt impact at frequencies higher than $20 \mathrm{GHz}$ becomes hardly distinguishable from the water relaxation.

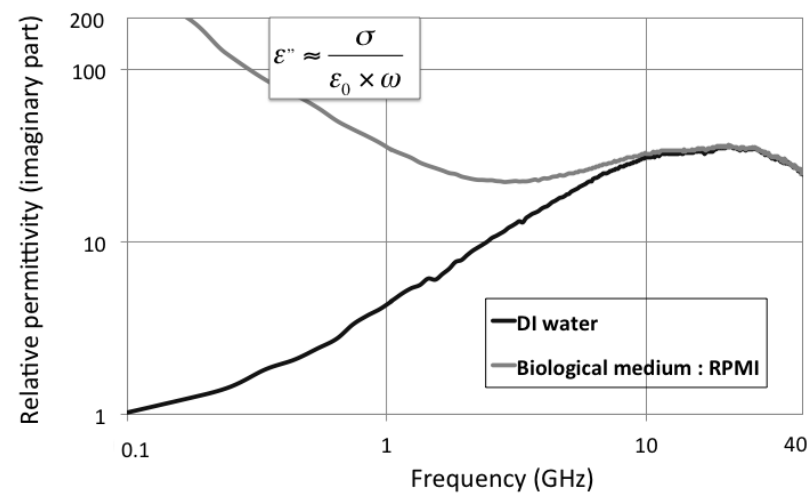

Fig. 6. Impact of salt content in the RPMI biological medium (added to $10 \%$ of Fœtal Calf Serum - FCS) compared to de-ionized water on the imaginary part of the relative permittivity.

This aspect of microwave sensing of cells in their culture medium enables to reuse the cells for further investigations after probing. This ability constitutes a major difference compared to the tools actually accessible by the biologists, such as the fluorescence-activated cell sorting (FACS) analyzers, which employ fluorescent tags, which may interfere with the living or modify it.

Being able to analyze cells in their culture medium also gives access to the temporal monitoring of cells in an appropriate living condition.

\section{Sensitivity and selectivity}

One main challenge for the microwave sensing resides in the sensitivity and selectivity of such technique to image (i.e. give bio-information) living cells. These capabilities and the enabled biological observations need to be precisely defined and further developed. Fulfilling this challenge will take time, as it implies to make the micro and millimeterwave circuit architectures progressively evolve.

The sensitivity and selectivity are under demonstration through different manners and at different levels :

○ with "in-flow" biological samples. It may constitute a better approach for large cells population analysis, in a similar way as it is currently done with commercial FACS. The required sub-millisecond analysis guides to narrowband techniques.

o with static biological samples (adherent or immobilized cells). These studies are suitable for broadband dielectric spectroscopy, which is mandatory for an accurate characterization of the biological matter and the understanding of the interaction between the electromagnetic waves with the living. They notably permit to define the most appropriate frequency ranges, suitable for cells and/or biomolecules discrimination.

High sensitivity at the single cell level has been demonstrated with interferometric microwave circuits, as schematically presented in Fig. 7 [45]-[46].

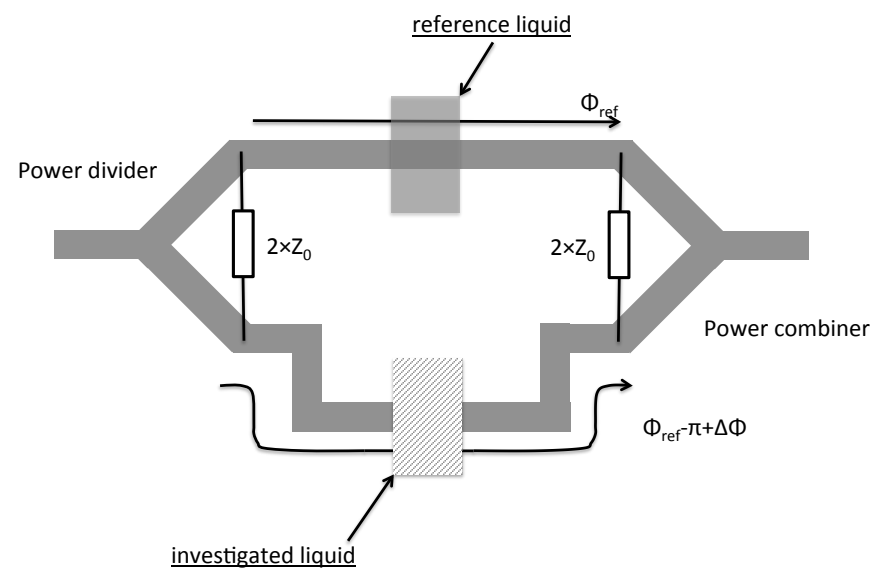

Fig. 7. Schematic view of an interferometric circuit developed for single cell analysis in flow.

With the power divider and combiner allied with two out of phase transmission paths, the circuit's readout reports the 
contrast between the reference and investigated liquids. As precise phase condition is required to reach high sensitivity, the operating bandwidth is consequently narrow.

Single frequency of $5 \mathrm{GHz}$ is exploited in [45] to discriminate the viability of yeast cells in water. The same principle with an alternative architecture operating at $1.6 \mathrm{GHz}$ in [46] also demonstrates high detection ability as $0.22 \mu \mathrm{m}$ cell's elevation is detected.

Selectivity at the cell and molecular levels represents a strategic challenge for microwave sensing. Such selective capability may enable the possible discrimination of cells phenotype, the understanding of this differentiation and then the comprehension of biological reactions and processes.

A recent investigation of biomolecules dissolved in water with microwave spectroscopy measurements [47] has demonstrated the possible identification of molecules due to the rheological and dielectric properties of each molecule, independently from their concentration. Such a differentiation is illustrated in Fig. 3, with the real and imaginary parts of the complex permittivity of two biomolecules in aqueous solution, the sugar saccharose and bovine albumin serum. For the same concentration, $100 \mathrm{mg} / \mathrm{ml}$ in this case, their respective microwave signature is significantly different. Applied to a wide variety of biomolecules, such as amino acids and carbohydrates, it is possible to establish a mapping of the dielectric properties of the biomolecules at a single frequency. The broadband dielectric spectroscopy of biomolecules leads to enormous data collection. The proposed dielectric mapping technique consequently facilitates the discrimination of molecules. This is illustrated in Fig. 8 with the clear identification of the biomolecules revealed by their respective contrasts in term of imaginary $\left(\Delta \varepsilon^{\prime \prime}\right)$ and real $\left(\Delta \varepsilon^{\prime}\right)$ parts of the permittivity with respect to de-ionized water, the host medium of the molecules.

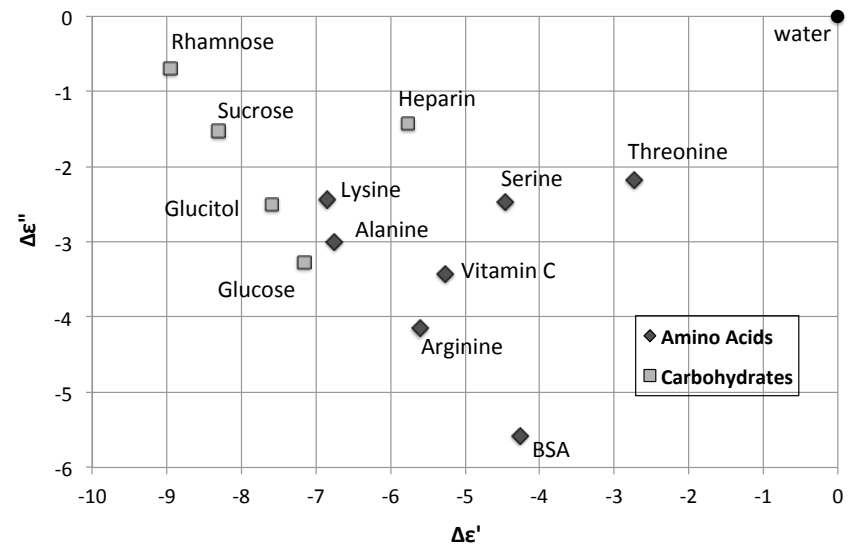

Fig. 8. Dielectric map at $20 \mathrm{GHz}$ of amino acids and carbohydrates in respect to de-ionized water as reference.

As far as cells are concerned, the dielectric spectroscopy of cells in liquid in the microwave range has been initiated at the micrometric scale by Facer et al. [26] with the measurements of hemoglobin solution and live E. coli bacteria up to 26.5
GHz. A similar structure as the one presented in Fig. 2 was used. Due to the large sensing area of the coplanar line, a large amount of cells in suspension (several millions of cells $/ \mathrm{ml}$ ) is required [48]. Toward miniaturization of the technique, an interdigitated capacitor configuration has been recently proposed and enables the dielectric spectroscopy of tens of cells in their traditional culture medium [49]. A photograph of this device is presented in Fig. 9. Its inset indicates around 70 RL lymphoma cells localized on top of the capacitor, whereas the Fig. 10 presents the capacitive contrast obtained for different concentrations of cells versus frequency.

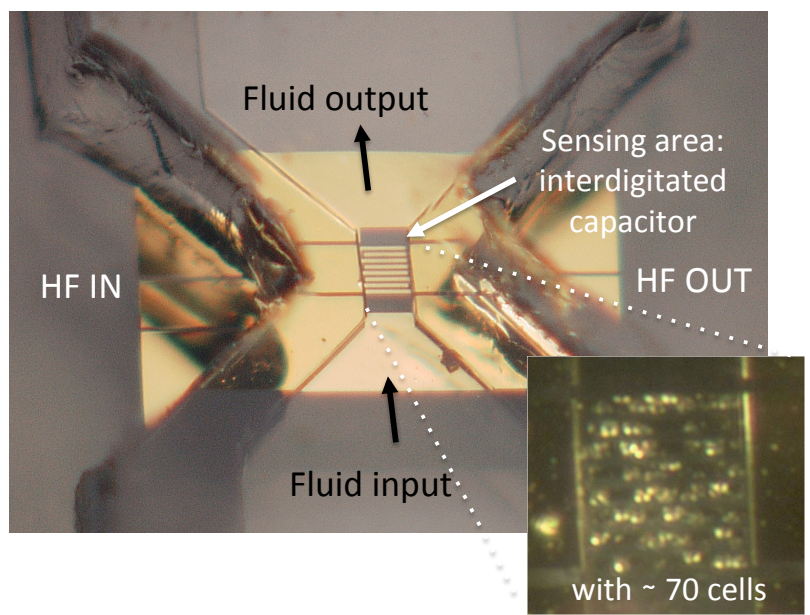

Fig. 9. Photograph of a microfluidic interdigitated capacitor [49]

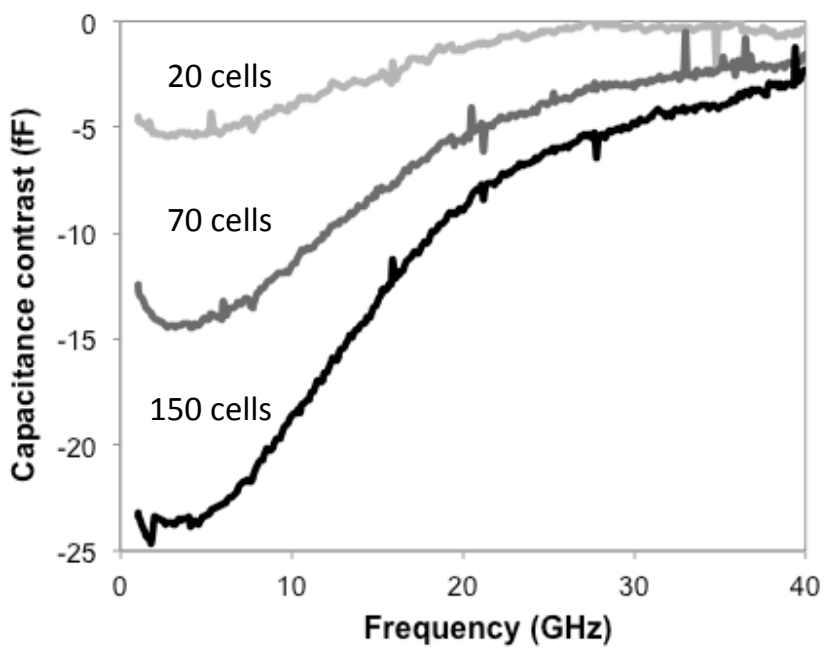

Fig. 10. Capacitive contrast of living RL lymphoma cells with respect to the host medium, RPMI with $10 \%$ of FCS, up to $40 \mathrm{GHz}$ for different cells concentrations: 20,70 and 150 cells. [49]

These results let us envision a possible cell counting and even the monitoring of proliferation. Considering the sensitivity level of $0.1 \mathrm{fF}$ obtained with such a sensor, the detection of a single cell in its culture medium, which means that no sample preparation or alteration is required, may be reached. This is confirmed by the detection in flow of a single yeast in water with a signal capacitive amplitude in the atto Farad range [45]-[46]. One important question then resides in the possible selectivity of the microwave sensing in term of 
cells pathological states and phenotypes.

The selectivity of dielectric properties has been established in the past with different tissues evaluation [13], [50]-[51] and with the possible discrimination of tumorous and nontumorous tissues [13], [16]-[20]. Tissues constitute however complex biological models with strong heterogeneity. An important variability due to adipose tissues has notably been defined through large investigations of breast cancer tissues [17].

The discrimination of living and dead cells in culture medium has been evaluated up to $40 \mathrm{GHz}$ with different circuit configurations: coplanar line [45]-[46], resonant structure [53]-[54] and interdigitated capacitor [49]-[55].

Fig. 11 presents the capacitive contrast of RL lymphoma cells for the two pathological states compared to their host medium. The capacitive contrast of the dead cells is lower than for the living ones. This may be explained by the fact that the dead cells exhibit pores into the membrane, which have favored the exchange of chemicals between the internal content of the cells and the external medium. Such a discrimination is already accessible by existing techniques but requires the addition of staining or fluorescent markers, which may interfere with the living. This is a crucial point for the microwave sensing: the developments of such a technique regarding the sensitivity and the selectivity have to be carried out with no compromise on the cells' non-invasivity.

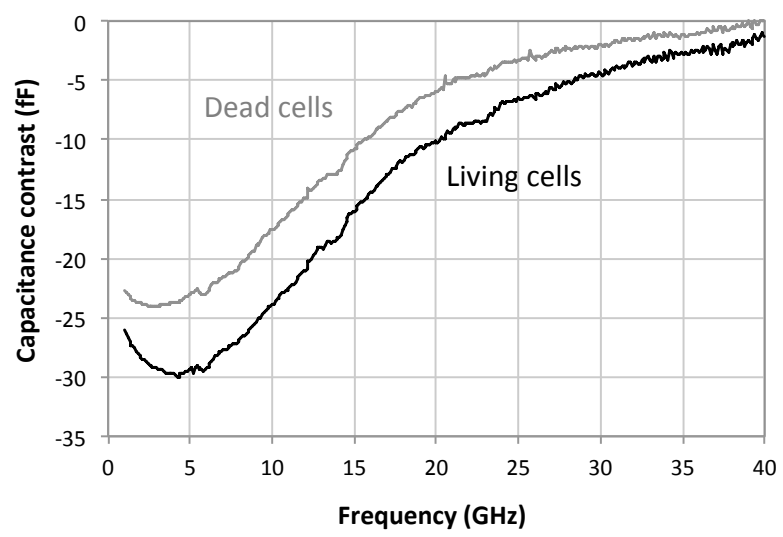

Fig. 11. Capacitive contrast of living and dead RL lymphoma cells with respect to the host medium, RPMI with $10 \%$ of BCS, up to $40 \mathrm{GHz}$. [55]

The above-mentioned references report on very distinct cells' type and pathological state, which is also the case for low-frequency impedance cytometry. This last technique, developed for more than a decade at the cellular level, has successfully demonstrated the differentiation of leukocytes [56]. However, as MHz-electromagnetic waves do not fully penetrate into the cells, fine phenotype discrimination cannot be achieved non-invasively [57]. On the contrary, GHz-regime is expected to overpass this limit.

Moreover, due to the intrinsic variability of biological and alive materials, and the heterogeneity within and across patients [20], specific attentions will have to be conducted to reach high repeatability and clarity in the observations. This remark is all the more important in the case of single cell analysis, as the measurements will also be confronted to the intrinsic living mechanisms such as the cell cycling notably, in the contrary of cells ensembles investigations, which inherently average the results.

\section{Real time monitoring for the investigations of biological reactions}

Among all the assets of the micro and millimeterwave sensing at the cellular level, a very important one consists in the real time monitoring of biological signatures, which means to look in real time at the impact of the environment on the cells due to therapeutic agents, drugs and other cells intervention.

To perform such investigations, strong circuits developments in compatibility with the microfluidic part are required.

Within this context, a preliminary study has been demonstrated in [58] using a reservoir placed on top of a coplanar line. The effect of DiMethylSulfOxide (DMSO) on the permittivity of a HEK-293 cells suspension has been evaluated over time. The results are presented in Fig. 12.

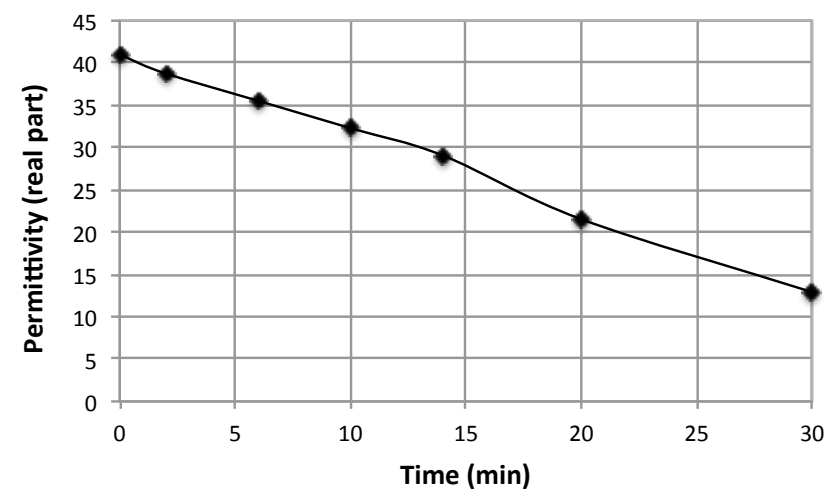

Fig. 12. Evolution over time of the permittivity of HEK-293 cells suspension submitted to $10 \%$ DMSO in PBS medium, derived from [58].

The DMSO acts as a cytotoxic solvent that deteriorates the cells membrane. The graph exhibits consequently a decrease on the permittivity over time, which reflects the impact on the cells. This first experiment is particularly important as it proves the ability of microwave sensing to probe in real time the response of a biological reaction. Questions remain about the sensitivity of the technique. Will the microwave sensing technique be sufficiently sensitive to distinguish cell variations? Which ones?

\section{CONCLUSION}

Microwave-based dielectric spectroscopy at the cellular and molecular levels is rapidly emerging. It benefits from the numerous researches already performed at the macroscale for cancer imaging and exhibits strong advantages. Recent 
researches already indicate that the technique permits to noninvasively analyze living cells in a liquid environment, and especially their culture medium. Further investigations are now necessary to further enhance the sensitivity, even if the single cell analysis is already reached, and to apply it to pertinent biological situations and processes. It will allow defining the limitations of the sensing technique, which has now to gain in maturity concerning the detection of very precise and weak biological modifications, like for instance in the cell cycle.

The fluorescent antibodies labeling techniques already available in commercial flow cytometers, as well as the staining microscopy, are extremely mature and present very high sensitivity and specificity. The microwave dielectric spectroscopy should consequently be developed in complete complementarity, providing an innovative way to observe the living at the microscale.

Foreseen applications are wide. Focusing on cancer investigations, the access to intracellular properties should enhance early diagnostic, facilitate anti-disease drugs screening due to the real time monitoring ability of the microwave spectroscopy, as well as personalized therapies and further biological mechanisms comprehension.

\section{REFERENCES}

[1] G.M. Whitesides, "The origins and the future of microfluidics," Nature, vol. 442, pp. 368-373, 2006.

[2] S. Grimmes, O. G. Martinsen, "Bioimpedance and bioelectricity basics," 2nd ed. Academic Press, 2008.

[3] X. Lu et. al. "Recent developments in single-cell analysis," Anal. Chim Acta, vol. 510, pp. 127-138, 2004.

[4] P.J. Hung et. al., "Continuous perfusion microfluidic cell culture array for high-throughput cell-based assays," Biotechnol. Bioeng., vol. 89, pp. $1-8,2005$.

[5] D. Wlodkowic, Z. Darzynkiewicz, "Microfluidics: emerging prospects for anti-cancer drug screening," World Journal of Clinical Oncology, vol. 1, no. 1, pp. 18-23, 2010.

[6] T. Sun, H. Morgan, "Single-cell microfluidic impedance cytometry: a review," Microfluid. Nanofluid., vol. 8, pp. 423-443, 2010

[7] H-B. Liu et. al., "Sensing minute change in biological cell monolayers with $\mathrm{THz}$ differential time-domain spectroscopy," Biosensors and Bioelectronics, vol. 22, pp. 1075-1080, 2007.

[8] A. Treizebré et. al., "Investigation of living cells with a $\mathrm{THz}$ BioMEMS," 5th Int. Conf. on Microtech. in Medicine and Biology, Quebec, Canada, 2009.

[9] N. Bao, J. Wang, C. Lu, "Recent advances in Electric Analysis of Cells in Microfluidic systems," Anal. Bioanal. Chem., vol. 391, pp. 933-942, 2008.

[10] A. Han, et. al., "Whole Cell Electrical Impedance Spectroscopy For Studying Ion Channel Activity", 13th Int. Conf. on Solid-Sate Sensor, Actuators and Microsystems proceedings, Seoul, Korea, June 5-9, 2005.

[11] Y.-B. Wang et. al., "A novel dual mode capacitor biosensor for realtime, label-free DNA detection," Int. Electron Devices Meeting, IEDM'06, 11-13 Dec. 2006.

[12] R. Bashir, "BioMEMS: state-of-the-art in detection, opportunities and prospects," Adv. Drug Delivery Rev., 56, pp.1565-1586, 2004.

[13] C. Polk and E. Postow, Handbook of Biological Effects of Electromagnetic Fields. CRC Press, 1996, ch. 1 by K.R. Foster and H.P. Schwan.

[14] A. Vander Vorst, A. Rosen, Y. Kotsuka, "RF/Microwave interaction with biological tissues," Wiley-Interscience, 2006.

[15] M.-R. Tofighi, A. Daryoush, "Comparison of two post-calibration correction methods for complex permittivity measurement of biological tissues up to $50 \mathrm{GHz}, "$ IEEE Trans. on Inst. and Meas., vol. 51, nº, pp. 1170-1176, 2002.

[16] P. Mehta, K. Chand, D. Narayanswamy, D.G. Beetner, R. Zoughi, W.V. Stoecker, "Microwave reflectometry as a novel diagnostic tool for detection of skin cancers," IEEE Trans. on Inst. and Meas., vol. 55, no. 4, pp. 1309-1316, Aug. 2006.

[17] M. Lazebnik, D. Popovic, L. McCartney, C.B. Watkins, M.J. Lindstrom, J. Harter, S. Sewall, T. Ogilvie, A. Magliocco, T.M. Breslin, W. Temple, D. Mew, J.H. Booske, M. Okoniewski, S.C. Hagness, "A large-scale study of the ultrawideband microwave dielectric properties of normal, benign and malignant breast tissues obtained from cancer surgeries," Phys. Med. Biol., vol. 52, pp. 6093-6115, 2007.

[18] E.C. Fear, P.M. Meaney, M.A. Stuchly, "Microwave for breast cancer detection?," IEEE Potentials, pp. 12-18, Feb/Mar. 2003

[19] N.K. Nikolova, "Microwave Imaging for Breast Cancer," IEEE Microwave Magazine, vol. 12, pp. 78-94, 2011

[20] L. Sha, E.R. Ward, B. Stroy, "A review of dielectric properties of normal and malignant breast tissue," Proceedings of IEEE SoutheastCon 2002 , pp. 457-462, 2002

[21] A. Lonappan, V. Thomas, G. Bindu, J. Jacob, C. Rajasekaran, K.T. Mathew, "New method of detecting lymphatic disease using microwaves," Microwave and Optical Technology Letters, vol. 49, no. 12, pp. 3166-3169, 2007.

[22] H. Fricke, "The electric resistance and capacity of blood for frequencies between 800 and 4.5 million cycles," J. Gen. Physiol., vol. 9, pp. 153$167,1925$.

[23] K.S. Cole and R.H. Cole, "Dispersion and absorption in dielectrics. I. Alternating current characteristics," J. of Chemical Physics, vol. 9, pp. 341-351, 1941

[24] G.J. Wilmink, J.E. Grundt, "Invited review article: Current state of research on biological effects of Terahertz radiation," J. Infrared Milli Terahz Waves, vol. 32, pp. 1074-1122, 2011.

[25] S.S. Stuchly, C.E. Bassey, "Microwave coplanar sensors for dielectric measurements," Meas. Sci. Technol., no. 9, 1998, pp. 1324-1329

[26] G.R. Facer et al., "Dielectric spectroscopy for bio-analysis: from $40 \mathrm{~Hz}$ to $26,5 \mathrm{GHz}$ in microfabricated waveguide," Applied Physic Letters, vol. 78, no. 7, pp. 996-998, Feb. 2001

[27] E.N. Shaforost, N. Klein, S.A. Vitusevich, A.A. Barannik, N.T. Cherpak, "High sensitivity microwave characterization of organic molecule solutions of nanoliter volume," APL, vol. 94, 112901, 2009.

[28] A.I. Gubin, AA. Barannik, N.T. Cherpak, S. Vitusevich, A. Offenhaeusser, N. Klein, "Whispering-gallery mode resonator technique for characterization of small volumes of biochemical liquids in microfluidic channel," IEEE European Microwave Conference Proceedings, Manchester, UK, pp. 615-618, 2011

[29] D.J. Rowe, A. Porch, D.A. Barrow, C.J. Allender, "Microfluidic device for compositional analysis of solvent systems at microwave frequencies," Sensors and Actuators B: Chemical, vol. 169, pp. 213-221, 2012

[30] J.C. Booth, J. Mateu, M. Janezic, J. Baker-Jarvis, J.A. Beall, "Broadband permittivity measurements of liquid and biological samples using microfluidic channels," IEEE MTT-S, pp. 1750-1753, 2006.

[31] K. Grenier and D. Dubuc et al., "Integrated Broadband Microwave and Microfluidic sensor dedicated to Bioengineering," IEEE Trans. on Microwave Theory and Techniques, vol. 57, Dec. 2009, pp. 3246-3253.

[32] J.C. Booth et al., "Quantitative permittivity measurements of nanoliter liquid in microfluidic channels to $40 \mathrm{GHz}$," IEEE Trans. on Instr. and Meas., vol. 59, no.12, 2010, pp. 3279-3288.

[33] J. El-Ali, P.K. Sorger, K.F. Jensen, "Cells on Chip", Nature, vol. 442, pp. 403-411, 2006.

[34] S.N. Kharkovsky, U.C. Hasar, "Measurement of mode patterns in a high-power microwave cavity," IEEE Trans. on Inst. and Meas., vol. 52, no. 6, pp. 1815-1819, Dec. 2003.

[35] W.J. Ellison, K. Lamkaouchi, J.-M. Moreau, "Water: a dielectric reference," J. of Molecular Liquids, vol. 68, pp. 171-279, 1996.

[36] R. Buchner, J. Barthel, J. Stauber, "The dielectric relaxation of water between $0^{\circ} \mathrm{C}$ and $35^{\circ} \mathrm{C}$," Chemical Physics Letters, vol. 306, pp. 57-63, 1999.

[37] W.J. Ellison, "Permittivity of pure water, at standard atmospheric pressure, over the frequency range $0-25 \mathrm{THz}$ and the temperature range $0-100^{\circ}$ C," J. Phys. Chem. Ref. Data, vol. 36, np. 1, pp. 1-18, 2007.

[38] J.-Z. Bao, C.C. Davis, M.L. Swicord, "Microwave dielectric measurements of erythrocyte suspensions," Biophysical Journal, vol. 66, pp. 2173-2180, 1994 
[39] H. Cao, V. Landge, U. Tata, Y. Seo, S. Rao, S. Tang, S. Spechler, J.-C. Chiao, , "An Implantable, Batteryless and Wireless Capsule with Integrated Impedance and $\mathrm{pH}$ Sensors for Gastroesophageal Reflux Monitoring," IEEE Trans. on Biomedical Engineering, vol. 99, 2012.

[40] M. Stacey, J. Stickley, P. Fox, C.O'Donnell, K. Schoenbach, S. Beebe, S. Buescher, "Increased cell killing and DNA damage in cells exposed to ultra-short pulsed electric fields," 2002 Annual Report Conference on Electrical Insulation and Dielectric Phenomena, pp. 79-82, 2002.

[41] V. Vojisavljevic, E. Pirogova, E. Cosic, "Low intensity microwave radiation as modulator of the L-lactate dehydrogenase activity," Med. Biol. Eng. Comput., vol. 49, pp. 793-799, 2011.

[42] P. Merola, C. Marino, G.A. Lovisolo, R. Pinto, C. Laconi, A Negroni, "Proliferation and apoptosis in a neuroblastoma cell line exposed to 900 $\mathrm{MHz}$ modulated radiofrequency field," Biolelectromagnetics, vol. 27, pp. 164-171, 2006.

[43] M. Liberti, F. Apollonio, C. Merla, G. d'Inzeo, "Microdosimetry in the microwave range: a quantitative assessment at single cell level," IEEE Antennas and Wireless Propagation Letters, vol. 8, pp. 865-868, 2009.

[44] F. Artis, C. Blatche, D. Dubuc, K. Grenier, "Biological Cells Proliferation in Microwave Microsystems," IEEE MTT-S, Montréal, Canada, June 2012.

[45] Y. Yang, H. Zhang, J. Zhu, G. Wang, T.-R. Tzeng, X. Xuan, K. Huang, P. Wang, "Distinguishing the viability of a single yeast cell with an ultra-sensitive radio frequency sensor," Lab on a Chip, vol. 10, pp. 553$555,2010$.

[46] G.A. Ferrier, S.F. Romanuik, D.J. Thomson, G.E. Bridges, M.R. Freeman, "A microwave interferometric system for simultaneous actuation and detection of single biological cells," Lab on a Chip, vol. 9, pp. 3406-3412, 2009.

[47] T.H. Basey-Fisher, S.M. Hanham, H. Andresen, S.A. Maier, M.M. Stevens, N.M. Alford, N. Klein, "Microwave Debye relaxation analysis of dissolved proteins: towards free-solution biosensing," $A P L$, vol. 99, 233703, 2011.

[48] D. Dubuc, K. Grenier, M. Poupot, J-J. Fournié, "Microwave signatures of Alive B-lymphoma cells suspensions," IEEE Radio and Wireless Symposium 2011 - Biowireless'11, Phoenix, USA, Jan. 2011.

[49] T. Chen, D. Dubuc, P. Poupot, J-J. Fournié, K. Grenier, "Accurate nanoliter liquid characterization up to $40 \mathrm{GHz}$ for biomedical applications: toward non-invasive living cells monitoring," IEEE Trans. on Microwave Theory and Techniques, vol. 60, ${ }^{\circ} 12$, pp. 4171-4177, Dec. 2012.

[50] S. Gabriel, R.W. Lau and C. Gabriel, "The dielectric properties of biological tissues", Physics in Medecine and Biology, vol. 41, no. 11, pp. 2231-2293, 1996

[51] H.P. Schwan, K. Li, "Capacity and conductivity of body tissues at ultrahigh frequencies," Proceedings of the I.R.E., pp. 1735-1740, 1953.

[52] H.P. Schwan, K.R. Foster, "RF-field interactions with biological systems: electrical properties and biophysical mechanisms," Proceedings of the IEEE, vol. 68, no. 1, 1980.

[53] K. Grenier, D. Dubuc, P-E. Poleni, M. Kumemura, T. Fujii, H. Toshiyoshi, H. Fujita, "Resonant based microwave biosensor for biological cells discrimination," IEEE Radio and Wireless Symposium 2010, New Orleans, USA, Jan. 2010

[54] L.Y. Zhang, C. Bounaix Morand du Puch, A. Lacroix, C. Dalmay, A. Pothier, C. Lautrette, S. Battu, F. Lalloué, M.-O. Jauberteau, P. Blondy, "Microwave biosensors for identifying cancer cell aggressiveness grade," IEEE MTT-S, Montréal, Canada, 2012.

[55] T. Chen, D. Dubuc, M. Poupot, J-J. Fournié, K. Grenier, "Broadband discrimination of living and dead lymphomas cells with a microwave interdigitated capacitor," IEEE Biowireless, Austin, USA, Jan. 2013.

[56] D. Holmes, D. Pettigrew, C.H. Reccius, J.D. Gwyer, C.van Berkel, J. Holloway, D.E. Davies, H. Morgan, "Leukocyte analysis and differentiation using high speed microfluidic single cell impedance cytometry," Lab on a Chip, vol. 9, pp. 2881-2889, 2009.

[57] D. Holmes, H. Morgan, "Single cell impedance cytometry for identification and counting of CD4 T-cells in human blood using impedance labels," Anal. Chem., vol. 82, pp. 1455-1461, 2010.

[58] S. Seo, T. Stintzing, I. Block, D. Pavlidis, M. Rieke, P.G Layer, "High frequency wideband permittivity measurements of biological substances using coplanar waveguides and application to cell suspensions," IEEE MTT-S, 2008, pp. 915-918.

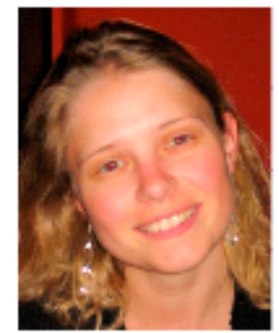

Katia Grenier (S'99, M'03) received her M.S. and $\mathrm{Ph} . \mathrm{D}$. degrees in electrical engineering from the University of Toulouse, Toulouse, France, in 1997 and 2000, respectively. She was engaged in microelectromechanical systems (MEMS) circuits on silicon. She was a Postdoctoral Fellow at Agere Systems (Bell Labs). In 2001, she joined the Laboratory of Analysis and Architecture of System of the National Scientific Research Center (LAAS-CNRS), Toulouse, France. From 2007 to 2009, she was with the Laboratory for Integrated Micromechatronic Systems CNRS (LIMMS-CNRS)/Institute of Industrial Science (IIS), Universtity of Tokyo, Tokyo, Japan, where she was engaged in launching research activities on microwave-based biosensors. Her research interests in LAAS-CNRS are now focused on the development of fluidicbased microsystems, notably for biological and medical applications at the cellular and molecular levels. Dr. Grenier is a member of the IEEE MTT-10 Technical Committee on Biological effect and medical applications of RF and microwave of the IEEE Microwave Theory and Techniques Society.

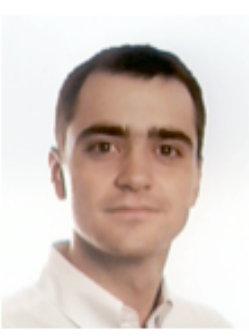

David Dubuc (S'99, M'03) received the Agregation degree from the Ecole Normale Supérieure de Cachan, Paris, France, in 1996, and the M.S. and $\mathrm{Ph} . \mathrm{D}$. degrees in electrical engineering from the University of Toulouse, Toulouse, France, in 1997 and 2001, respectively. Since 2002, he has been an Associate Professor with the University of Toulouse, and a Researcher with the Laboratory of Analysis and Architecture of System part of National Scientific Research Center (LAAS-CNRS), Toulouse, France. From 2007 to 2009, he was a Visiting Senior Researcher with the Laboratory of Integrated Micromechatronic Systems (LIMMS-CNRS)/Institute of Industrial Science (IIS), University of Tokyo, Tokyo, Japan. His research interests include the development of microwave circuits integrated due to microtechnologies and their application to wireless telecommunication and biology.

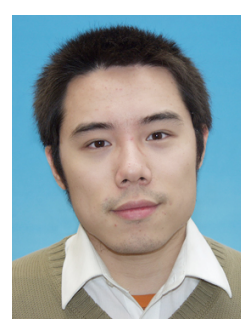

Tong Chen received the M.S. degree in electrical engineering from the University of Toulouse, Toulouse, France, in 2009. He is currently working toward the Ph.D. degree from the University of Toulouse, at the Laboratory of Analysis and Architecture of System part of National Scientific Research Center (LAAS-CNRS), Toulouse, France. His research interests involve the development of miniature microwave-based biosensors for dielectric spectroscopy.

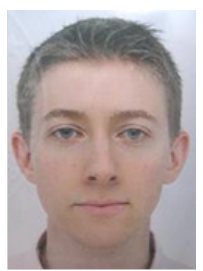

François Artis received the M.S. degree in electrical engineering from the University of Toulouse, Toulouse, France, in 2011. He is currently working toward the Ph.D. degree from the University of Toulouse, at the Laboratory of Analysis and Architecture of System part of National Scientific Research Center (LAAS-CNRS), Toulouse, France. His work deals with the characterization of human biological cells by dielectric spectroscopy.

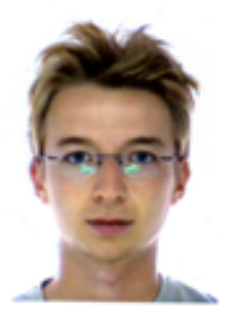

Thomas Chretiennot graduated from the Ecole Nationale Superieure de l'Electronique et de ses Applications (E.N.S.E.A.), Cergy, France, in 2009, a French graduate school in electrical engineering, computer science and telecommunications. $\mathrm{He}$ is currently working towards his $\mathrm{PhD}$ degree at the Laboratory of Analyse and Architecture of System part of National Scientific Research Center (LAASCNRS), Toulouse, France. His work deals with the development of microwave and microfluidic 


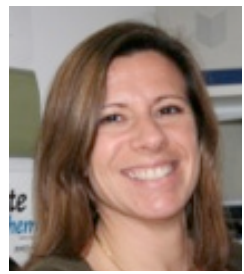

Mary Poupot received her M.R. and PhD degrees in Biochemistry from the University of Paul Sabatier, Toulouse, France, in 1993 and 1997, respectively. From 2001 to 2007 she was a Postdoctoral Fellow at the Center of Physiopathology of Toulouse Purpan, Toulouse, France. She was interested in the activation of human lymphoid cells to eradicate cancer cells. Since 2007, she has been a Researcher at the Cancer Research Center of Toulouse. Now, her research interests are based on the impact of the tumor microenvironment on the survey of cancer cell in particular in hematopoietic diseases.

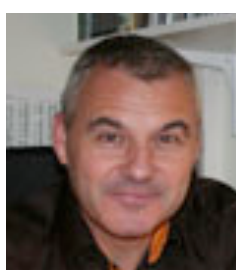

Jean-Jacques Fournié received her M.R. and $\mathrm{PhD}$ degrees in Biochemistry from the University of Paul Sabatier, Toulouse, France, in 1983 and 1986, respectively. From 1988 to 1989 he was a Postdoctoral Fellow Post-doc fellowship at CIRC (currently Garvan Institute-), Sydney Univ. Australia. Since 1986 he has been a Researcher and in 1990, he settled a cell immunology group focussed on nonpeptide antigens activating $\mathrm{T}$ cells at the the Center of Physiopathology of Toulouse Purpan, Toulouse, France. In 1999, he co-founded the corporate company Innate Pharma (Marseille, France) producing new anticancer immunotherapeutics. Since 2011, he has been Director of the Cancer Research Center of Toulouse, France and the aim of his team is based on the immunetargeting of hematopoietic diseases. 